\title{
The efficacy and safety of lenalidomide in the treatment of multiple myeloma patients after allo-hematopoietic stem-cell transplantation: a systematic review and meta-analysis
}

\author{
Jiansheng Zhong ${ }^{1}$, Xing Zhang ${ }^{1}$, Minyan Liu ${ }^{2}$ \\ ${ }^{1}$ Department of Hematology, Guangzhou Red Cross Hospital, Jinan University, Guangzhou, China; ${ }^{2}$ Emergency Department, Sun Yat-sen Memorial \\ Hospital, Sun Yat-sen University, Guangzhou, China \\ Contributions: (I) Conception and design: J Zhong; (II) Administrative support: J Zhong; (III) Provision of study materials or patients: J Zhong, X \\ Zhang, M Liu; (IV) Collection and assembly of data: J Zhong; (V) Data analysis and interpretation: J Zhong; (VI) Manuscript writing: All authors; (VII) \\ Final approval of manuscript: All authors. \\ Correspondence to: Jiansheng Zhong. Department of Hematology, Guangzhou Red Cross Hospital, 396 Tong Fu Zhong Road, Guangzhou 510220, \\ China. Email: zhongjs21@163.com.
}

Background: There has been long-standing controversy regarding the effectiveness and safety of
lenalidomide as a maintenance treatment for patients with multiple myeloma $(M M)$ after allogeneic cell
transplantation. This meta-analysis aimed to explore the effectiveness and safety of lenalidomide in the
maintenance treatment of $M M$ patients after allogeneic cell transplantation based on published data.

Methods: A systematic review and meta-analysis was conducted in English and Chinese databases, covering all available publications until 1 December 2020. Statistical analysis was performed using the software STATA 14.0, and odds ratios (ORs) combined with 95\% confidence intervals (CIs) were calculated to explore the efficacy and safety of lenalidomide in the treatment of MM patients after allogeneic cell transplantation.

Results: A total of 173 MM cases in 8 independent studies from 2007 to 2014 were included. Through a single-arm meta-analysis of the disease status of $M M$ patients after lenalidomide treatment, $3.6 \%$ of patients were in minimal response ( $M R, P=0.006), 39.0 \%$ were in complete remission $(C R, P=0.169), 20.2 \%$ in partial remission ( $\mathrm{PR}, \mathrm{P}<0.001), 12.8 \%$ in very good partial remission (VGPR, $\mathrm{P}=0.049)$, and $9.7 \%$ in $\mathrm{SD}(\mathrm{P}=0.023)$; the $\mathrm{PD}$ was $5.6 \%(\mathrm{P}=0.010)$. Through meta-analysis of adverse reactions after taking lenalidomide, $35.3 \%$ $(\mathrm{P}=0.628)$ of participants developed acute graft-versus-host disease (GVHD); $22.6 \%(\mathrm{P}=0.049)$ developed chronic GVHD; 20.3\% ( $\mathrm{P}=0.001)$ developed infection; $22.5 \%(\mathrm{P}=0.352)$ had thrombocytopenia; $32.5 \%$ $(\mathrm{P}<0.000)$ had neutropenia; pain occurred in $17.8 \%(\mathrm{P}=0.350)$ of patients, and peripheral neuropathy occurred in $17.8 \%(\mathrm{P}=0.995)$ of participants. The overall survival (OS) of $\geq 2$ years and progression-free survival (PFS) of $\geq 2$ years of MM patients after allo-hematopoietic-stem-cell transplantation (HSCT) taking lenalidomide were analyzed, and the results were 64.9\% ( $\mathrm{P}=0.049)$ and 58.4\% ( $\mathrm{P}=0.890)$, respectively.

Discussion: Lenalidomide is effective in the treatment of MM patients after allo-HSCT, and reducing the incidence of infection and peripheral neuropathy, but it is not effective in reducing GVHD and blood system adverse reactions.

Keywords: Lenalidomide; multiple myeloma (MM); allo-HSCT; meta-analysis

Submitted Apr 30, 2021. Accepted for publication Jul 19, 2021.

doi: 10.21037/apm-21-1598

View this article at: https://dx.doi.org/10.21037/apm-21-1598 


\section{Introduction}

Multiple myeloma (MM) is a malignant plasma cell disease characterized by abnormal proliferation of bone marrow plasma cells accompanied by excessive production of monoclonal immunoglobulin or light chain (M protein). Very few patients express the unsecreted type of MM, and may not produce $M$ protein. MM is often accompanied by multiple osteolytic damage, hypercalcemia, anemia, and kidney damage (1), and various bacterial infections are also prone to occur due to the inhibition of the production of normal immunoglobulin.

Accounting for about $10 \%$ of hematological malignancies, $\mathrm{MM}$ is the second most common tumor of the hematological system (2), mostly affects the middleaged and elderly, and currently there is no complete cure. In the 1970's and 1980's, MM was treated with traditional chemotherapy such as melphalan and prednisone (MP) or dexamethasone (VAD), and the complete remission (CR) rate was only 3-5\% (3). From the 1980's to the 1990's, due to the rise of auto-HSCT and allo-hematopoietic-stemcell transplantation (HSCT) technologies, the efficacy of MM treatment was significantly improved. Among them, the allo-HSCT technique is an alternative method when traditional chemotherapy and auto-HSCT cannot be effectively applied due to its graft anti-tumor effect (graft versus myeloma, GVM), but the higher incidence of graft-versus-host disease (GVHD) limits its widely used in clinical practice. Lenalidomide is a structural analog of thalidomide, and is a new generation immunomodulator developed by Celgene Corp. (Summit, NJ, USA). It is mainly used for the treatment of MM, myelodysplastic syndrome, and lymphoma. Lenalidomide is commonly used in the treatment of MM patients who have previously received allo-HSCT due to its unique immune regulation and tumor-killing dual mechanism; however, there are still clinical controversies regarding its effectiveness and safety. Therefore, we conducted a single-arm meta-analysis on the effectiveness and safety of lenalidomide in the treatment of MM patients after allo-HSCT. We present the following article in accordance with the PRISMA reporting checklist (available at https://dx.doi.org/10.21037/apm-21-1598).

\section{Methods}

This meta-analysis was conducted with guidance from the Cochrane handbook and reported according to the Preferred Reporting Items for Meta-Analyses (PRISMA) guidelines. The process followed a priori established protocol.

\section{Data sources and search strategy}

Relevant publications were identified by an electronic literature search of PubMed (Medline), EMBASE, Web of Science, Cochrane Library, Chinese National Knowledge Infrastructure (CNKI) Database, Wanfang, and Weipu Databases up to 1 December 2020. The search was conducted by an experienced medical librarian, with input from the study's investigators, and it included the following keywords: 'Revimid' or 'Revlimid' or 'CC-5013' or 'CC5013' or 'IMiD3 Cpd', or 'Multiple Myelomas' or 'Myelomas, Multiple' or 'Myeloma, Multiple' or 'Myeloma, Plasma-Cell' or 'PlasmaCell Myeloma' or 'Myelomatosis' or 'Plasma Cell Myeloma'or 'Cell Myeloma, Plasma' or 'Kahler Disease' or 'MyelomaMultiple' or 'Myeloma Multiple'or 'MM' or 'Transplantation' or 'Transplantations' or 'Cell Transplants' or 'Transplant, Cell' or 'Transplants, Cell'or 'allogene'. Medical Subject Headings (MeSH) terms were also searched: 'Lenalidomide' or 'allogeneic cell transplantation' or 'multiple myeloma'. Due to language limitations, only English and Chinese documents were reviewed.

\section{Study selection}

Cohort, cross-sectional, and case-control studies were deemed as acceptable through the study selection process. The inclusion criteria were as follows: (I) studies focused on patients with MM; (II) participants received allogeneic cell transplantation; (III) participants received lenalidomide treatment; (IV) number of participants was no less than 5; and (V) the disease progression after lenalidomide treatment was reported, including adverse reactions, progression-free survival (PFS), and overall survival (OS) data. The exclusion criteria were as follows: (I) duplicate literature, (II) data required for analysis was not reported, (III) studies only including infants (aged $<6$ years old); (IV) studies lacking adequate sample size (less than 5 participants with $M M$ ); and (V) animal studies. In situations where studies contained substantial overlapping data from the same patient population ( $>2$ years of overlapping data), only data from the most comprehensive study (greatest number of participants) was included. In studies with a minor degree of overlap ( $<2$ years overlap), both datasets were included with a pre-planned sensitivity analysis. 


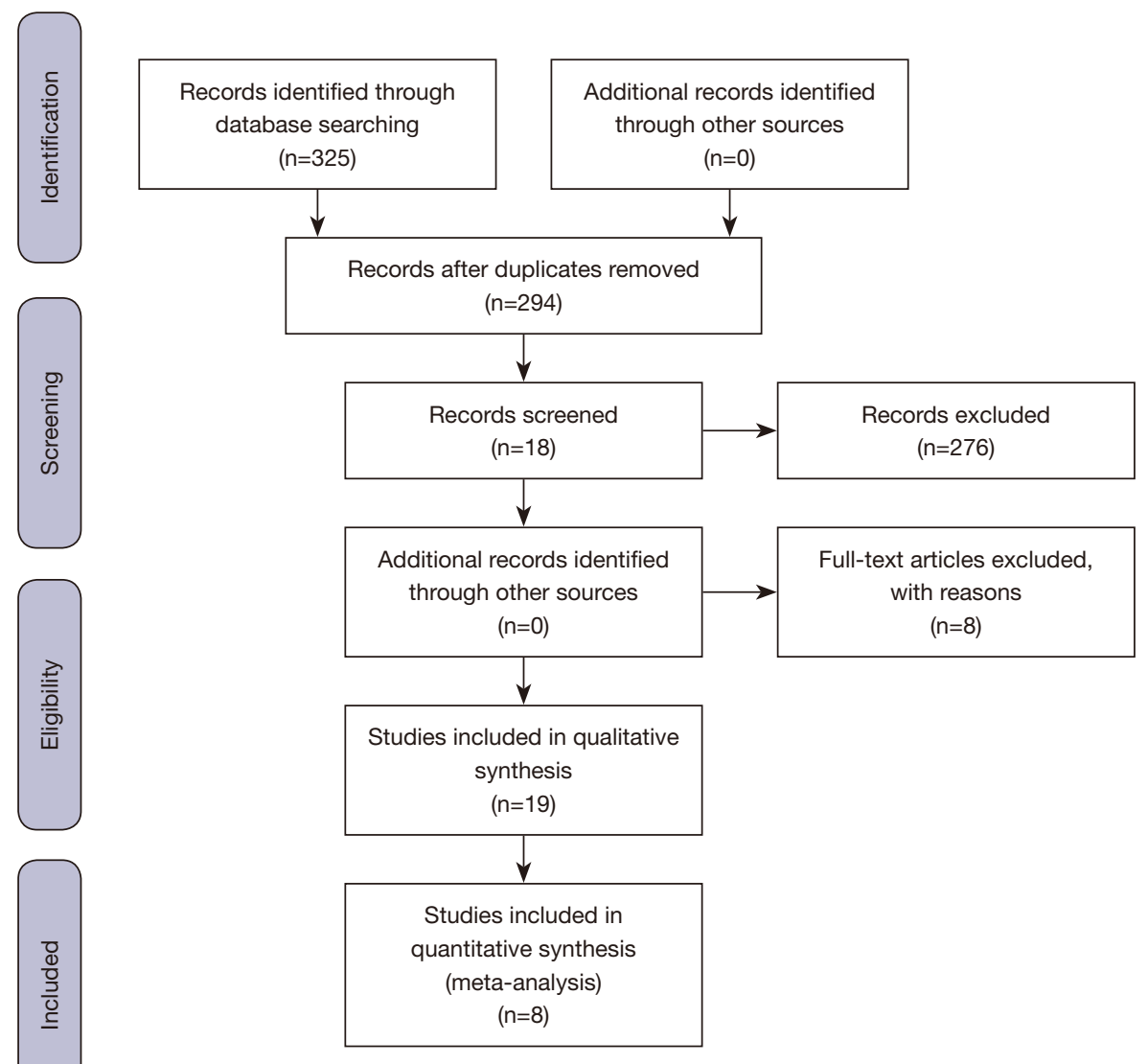

Figure 1 Flow chart depicting search strategy for the systematic review.

\section{Data extraction and quality assessment}

Data extraction was performed independently by 2 investigators and confirmed by the third investigator. The data extraction process included: (I) study characteristics (primary author, year of publication/time period of study, and study design); (II) characteristics of participants and MM (number of cases, participant age). For this study, the methodological quality of the included studies was assessed using the Quality Assessment of Diagnostic Accuracy Studies-2 (QUADAS-2) Scale. Studies were scored across 4 categories: random sequence generation (3 questions), randomized hiding (4 questions), blind method (3 questions), and quitting and withdrawal (2 questions). Study quality was defined as low and high corresponding to scores of $1-3$ and $4-7$, respectively.

\section{Statistical analysis}

The statistical software Stata 14.0 (StataCorp. LLC.,
College Station, TX, USA) was used to meta-analyze the extracted data. Before the meta-analysis, the heterogeneity between studies was analyzed by chi-square $\left(\chi^{2}\right)$ test. When $\mathrm{P}<0.05$, heterogeneity was considered significant. Then, the random effects model was used to merge and the cause of heterogeneity was further analyzed. When $\mathrm{P}>0.05$, there was no significant heterogeneity, and the fixed effects model was used to merge data. The correlation index was expressed by odds ratio (OR) and $95 \%$ confidence interval (CI). A P value $<0.05$ was considered statistically significant.

\section{Results}

\section{Results of document search}

As shown in Figure 1, a total of 325 records were identified after the initial search of databases. After reading the title/ abstract, 307 records were excluded via removal of duplicates or irrelevant studies. The remaining 8 records were further analyzed by full-text reading, resulting in the exclusion of 
1 record according to the inclusion and exclusion criteria. Ultimately, after qualitative and quantitative data synthesis, only 8 independent studies, including 7 cohort studies and 1 case-control study, were included in this meta-analysis, with a total of 181 participants.

\section{Characteristics of included studies}

All of the included 8 articles were published between 2009 and 2014, of which 2 were published in Germany, 2 in the USA, 2 in the Netherlands, and 2 in the USA. All of the participants were Caucasians, aged $48-58.5$ years old. The characteristics of included studies are summarized in Table 1.

\section{Disease status after lenalidomide treatment}

Firstly, we conducted a single rate meta-analysis of the disease progression of MM patients after receiving lenalidomide treatment. Among them, 2 studies reported that the condition after treatment with lenalidomide was minimal response (MR), and the overall rate was 3.6\% (CI: 0.004 to $0.097, \mathrm{P}=0.006$ ), 8 studies reported that the condition was $\mathrm{CR}$, and the overall rate was $39.0 \%$ (CI: 0.303 to $0.480, \mathrm{P}=0.169), 6$ studies reported that the condition was partial remission (PR), and the overall rate was $20.2 \%$ (CI: 0.092 to $0.343, \mathrm{P}<0.001$ ), and 7 studies reported that the participants' condition was very good partial remission (VGPR), with an overall rate of $12.8 \%$ (CI: 0.066 to 0.208 , $\mathrm{P}=0.049)$. A total of 6 studies reported that the participants' condition after treatment with lenalidomide was stable disease (SD) with an overall rate of $9.7 \%$ (CI: 0.040 to 0.175 , $\mathrm{P}=0.023)$, and 3 studies reported that the condition was progressive disease (PD), with an ORR of 5.6\% (CI: 0.013 to $0.124, \mathrm{P}=0.010$ ) (Figure 2, Table 2).

\section{The statistics of adverse events}

We conducted a single rate meta-analysis of the adverse reactions of MM patients after allogeneic cell transplantation after receiving lenalidomide treatment. A total of 8 studies reported the incidence of acute GVHD in participants after lenalidomide treatment, with an overall rate of $35.3 \%$ (CI: 0.290 to $0.419, \mathrm{P}=0.628$ ). A total of 4 studies reported the incidence of chronic GVHD with an overall rate of $22.6 \%$ (CI: 0.109 to $0.370, \mathrm{P}=0.059$ ); 3 studies reported infections after treatment, and the overall rate was $20.3 \%$ (CI: 0.031 to $0.471, \mathrm{P}=0.001)$. There were 5 studies that simultaneously reported the incidence of thrombocytopenia and neutropenia, the overall rates were $22.5 \%$ (CI: 0.157 to $0.302, \mathrm{P}=0.352$ ) and $32.5 \%$ (CI: 0.110 to $0.590, \mathrm{P}<0.001$ ), respectively. There were 3 that reported the symptoms of pain in patients lenalidomide treatment with an overall rate of $17.8 \%$ (CI: 0.092 to $0.284, \mathrm{P}=0.350$ ). In addition, there were 3 studies which reported the occurrence of peripheral neuropathy, and the rate was $17.8 \%$ (CI: 0.109 to $0.256, \mathrm{P}=0.995$ ) (Figures 3-9, Table 2)

\section{Survival}

We performed a one-arm meta-analysis of PFS and OS in MM patients who had been treated with lenalidomide. There were 5 studies which included participant PFS ( $\geq 2$ years) data, and the analysis results showed that the overall rate was $58.4 \%$ (CI: 0.499 to $0.667, \mathrm{P}=0.890$ ). In addition, all 8 studies mentioned participants' OS ( $\geq 2$ years) data and analysis showed that the pooled rate of OS ( $\geq 2$ years) in MM patients who had received allogeneic cell transplantation after lenalidomide treatment was $64.9 \%$ (CI: 0.451 to $0.824, \mathrm{P}<0.001$ ) (Figures 10,11 , Table 2).

\section{Discussion}

The disease MM is a malignancy of antibody-secreting, terminally differentiated B cells that home to and expand in the bone marrow, with symptoms related to anemia, immunosuppression, bone destruction, and renal failure. Allo-HSCT is widely used clinically as an effective method for the treatment of MM. However, there are many adverse reactions after allo-HSCT, such as GVHD, infection, thrombocytopenia, pain, and so on, which will cause serious distress to patients' postoperative life, and even affect their MM treatment. As the 4-amino-glutaryl derivative of thalidomide, lenalidomide is commonly used in the treatment of various malignant hematological diseases and solid tumors due to its reduced side effects and unique dual mechanism of immune regulation and tumor killing. There is no relevant report at present regarding whether lenalidomide can effectively improve the treatment effect of MM patients after allo-HSCT and reduce the occurrence of adverse reactions; thus, we conducted this meta-analysis.

We first analyzed the disease status of $173 \mathrm{MM}$ patients who received allo-HSCT after taking lenalidomide. The results showed that the comprehensive CR ratio reached 46.6\% (CI: $30.1 \%$ to $63.3 \%$ ), and the PR and VGPR also reached $21.8 \%$ (CI: $12.8 \%$ to $32.5 \%$ ) and $14.7 \%$ (CI: $9.7 \%$ to $20.7 \%$ ), respectively, while the comprehensive 


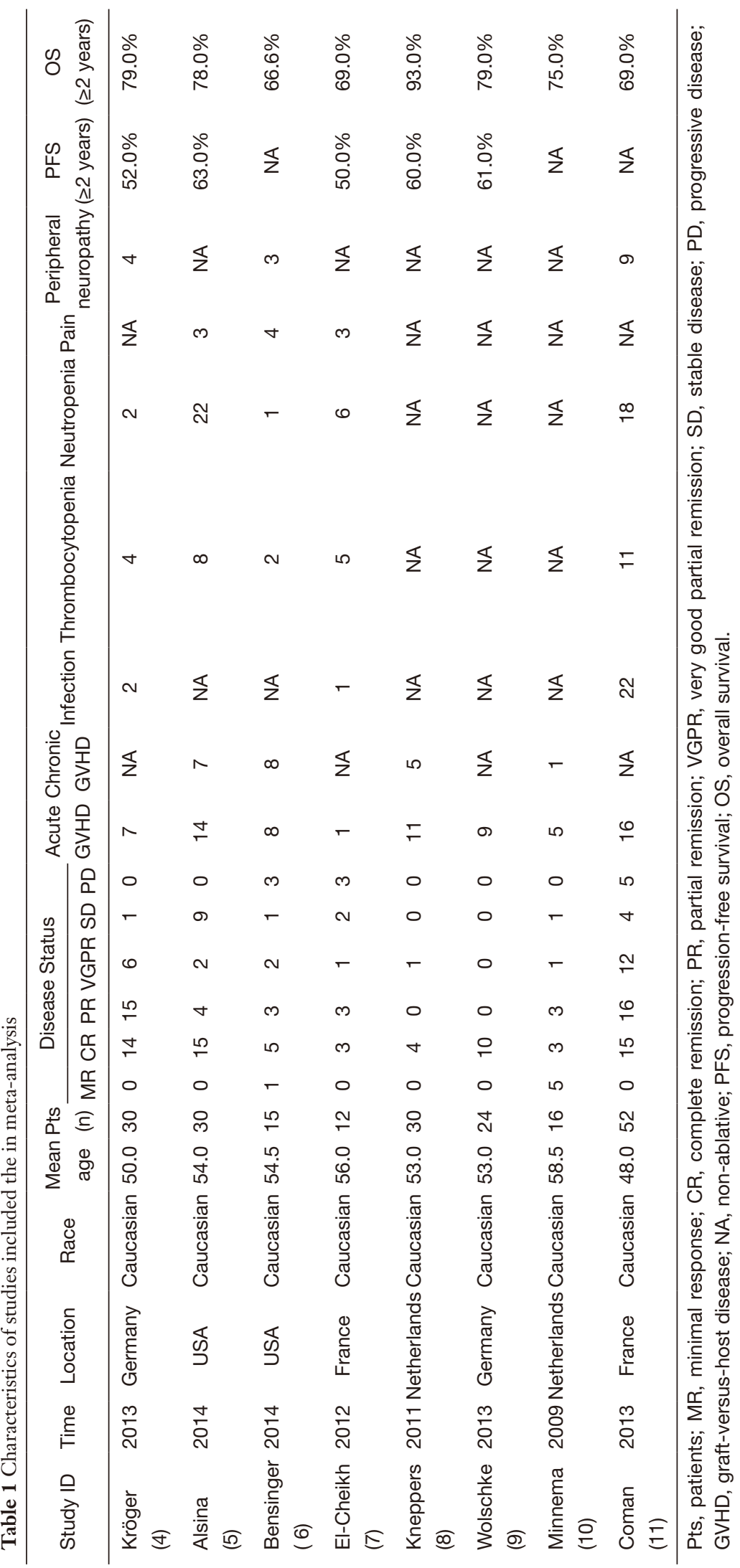







Table 2 Summary table of meta-analysis results

\begin{tabular}{|c|c|c|c|c|}
\hline Items & Overall rate & $95 \% \mathrm{Cl}$ & $P$ value & $\mathrm{I}^{2}$ \\
\hline MR & 0.036 & $0.004-0.097$ & 0.006 & $64.40 \%$ \\
\hline $\mathrm{CR}$ & 0.390 & $0.303-0.480$ & 0.169 & $79.10 \%$ \\
\hline PR & 0.202 & $0.092-0.343$ & 0.000 & $57.60 \%$ \\
\hline SD & 0.097 & $0.040-0.175$ & 0.023 & $48.00 \%$ \\
\hline PD & 0.056 & $0.013-0.124$ & 0.010 & $60.50 \%$ \\
\hline \multicolumn{5}{|l|}{ Adverse events } \\
\hline Acute GVHD & 0.353 & $0.290-0.419$ & 0.628 & $0.00 \%$ \\
\hline Thrombocytopenia & 0.225 & $0.157-0.302$ & 0.352 & $9.60 \%$ \\
\hline Neutropenia & 0.325 & $0.110-0.590$ & 0.000 & $89.90 \%$ \\
\hline Pain & 0.178 & $0.092-0.284$ & 0.350 & $4.80 \%$ \\
\hline Peripheral neuropathy & 0.178 & $0.109-0.256$ & 0.995 & $0.00 \%$ \\
\hline \multicolumn{5}{|l|}{ Survival } \\
\hline PFS ( $\geq 2$ years) & 0.584 & $0.499-0.667$ & 0.890 & $0.00 \%$ \\
\hline OS ( $\geq 2$ years) & 0.649 & $0.451-0.824$ & 0.049 & $82.00 \%$ \\
\hline
\end{tabular}

The numerical results of overall rate and $95 \% \mathrm{Cl}$ are obtained after double sine transformation. $\mathrm{Cl}$, confidence interval; MR, minimal response; CR, complete remission; PR, partial remission; VGPR, very good partial remission; SD, stable disease; PD, progressive disease; GVHD, graft-versus-host disease; PFS, progression-free survival; OS, overall survival.


Figure 3 A meta-analysis of acute GVHD in patients with MM treated with lenalidomide. (A) Forest diagram and (B) Funnel diagram are the results of the analysis of the prevalence of acute GVHD. MM, multiple myeloma; GVHD, graft-versus-host disease.

PD ratio was only $6 \%$ (CI: $1.5 \%$ to $13.2 \%$ ), indicating that lenalidomide is effective and has a good performance. To address safety concerns, we analyzed the incidence of a series of possible complications. Among them, the most commonly occurring were acute GVHD and chronic GVHD in patients after allo-HSCT, at rates of $35.3 \%$ (CI: $29 \%$ to $41.9 \%$ ) and $22.6 \%$ (CI: $10.9 \%$ to $37 \%$ ), respectively; there was no effect in reducing the incidence 

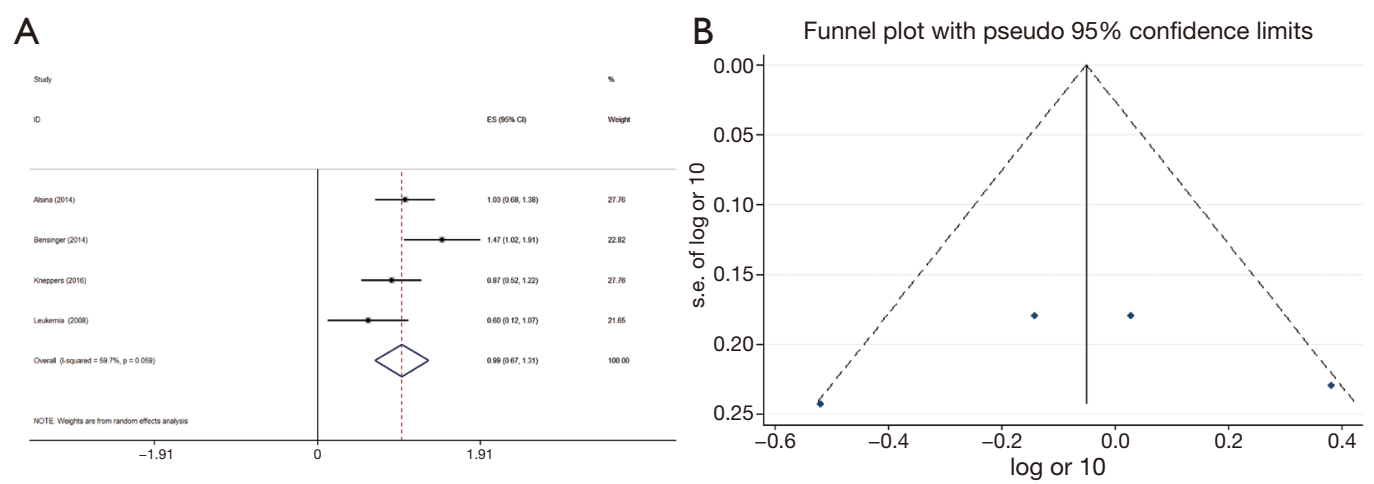

Figure 4 A meta-analysis of chronic GVHD in patients with MM treated with lenalidomide. (A) Forest diagram and (B) Funnel diagram are the results of the analysis of the prevalence of chronic GVHD. MM, multiple myeloma; GVHD, graft-versus-host disease.
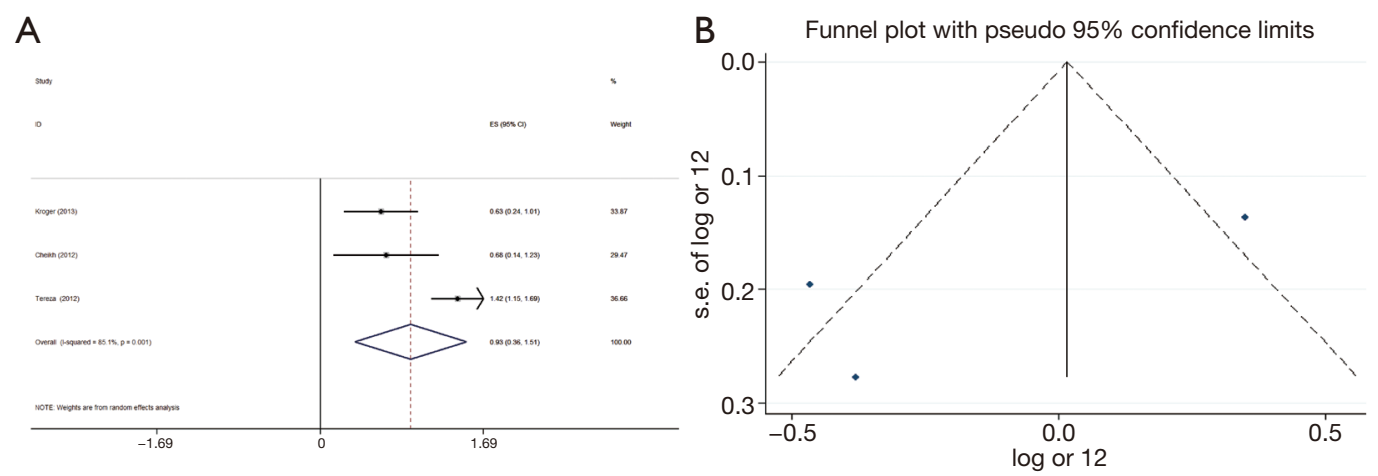

Figure 5 A meta-analysis of infections in patients with $M M$ treated with lenalidomide. (A) Forest diagram and (B) Funnel diagram are the analysis results of infection prevalence. MM, multiple myeloma.
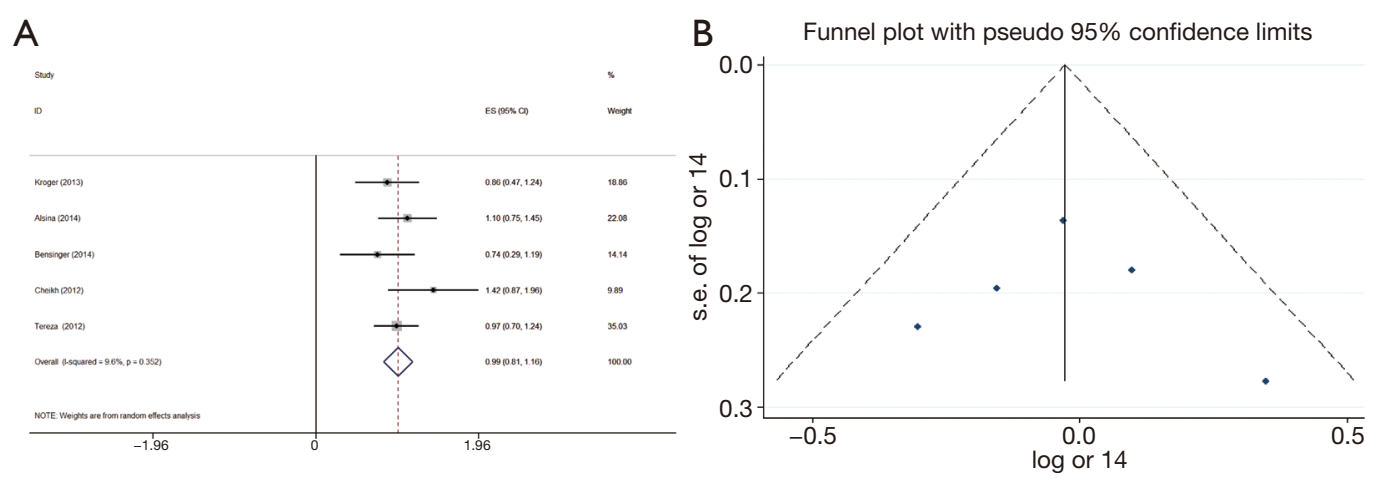

Figure 6 A meta-analysis of thrombocytopenia in patients with MM treated with lenalidomide. (A) Forest diagram and (B) Funnel diagram are the results of the analysis of the prevalence of thrombocytopenia. MM, multiple myeloma.

of GVHD. In addition, the overall incidence of infection after treatment was $20.3 \%$ (CI: $3.1 \%$ to $47.1 \%$ ), which was at a low level. The main adverse reactions in the blood system after taking lenalidomide were thrombocytopenia
(22.5\%, CI: $15.7 \%$ to $30.2 \%)$ and neutropenia (32.5\%, CI: $11.0 \%$ to $59.0 \%$ ), and $17.8 \%$ of participants experienced pain. The incidence of peripheral neuropathy was $17.8 \%$ (CI: $10.9 \%$ to $25.6 \%$ ), which was somewhat improved 

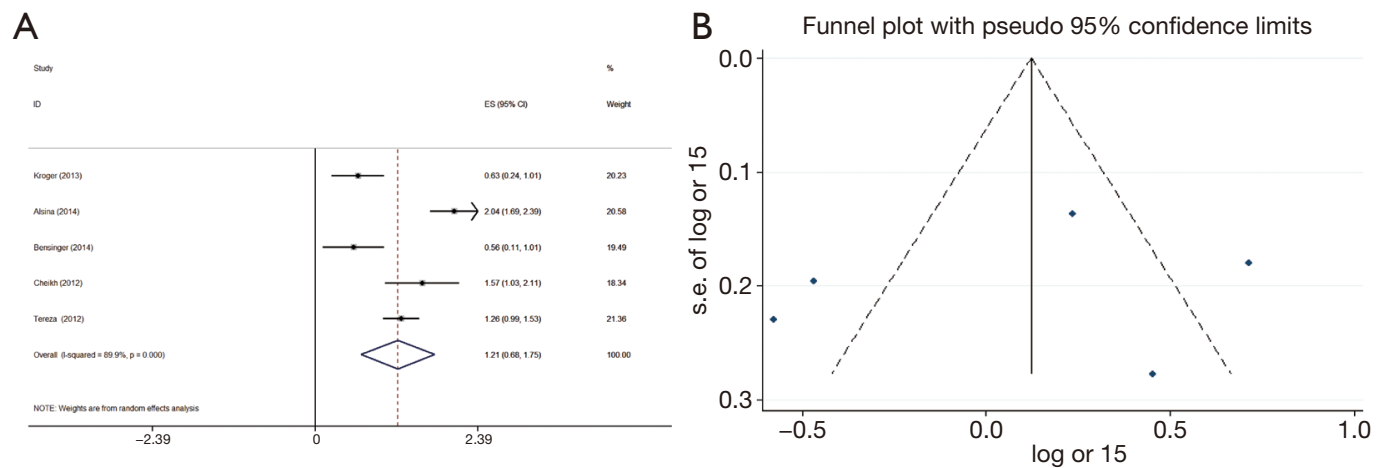

Figure 7 A meta-analysis of neutropenia in patients with MM treated with lenalidomide. (A) Forest diagram and (B) Funnel diagram are the results of the analysis of the prevalence of neutropenia in patients with MM treated with lenalidomide. MM, multiple myeloma.
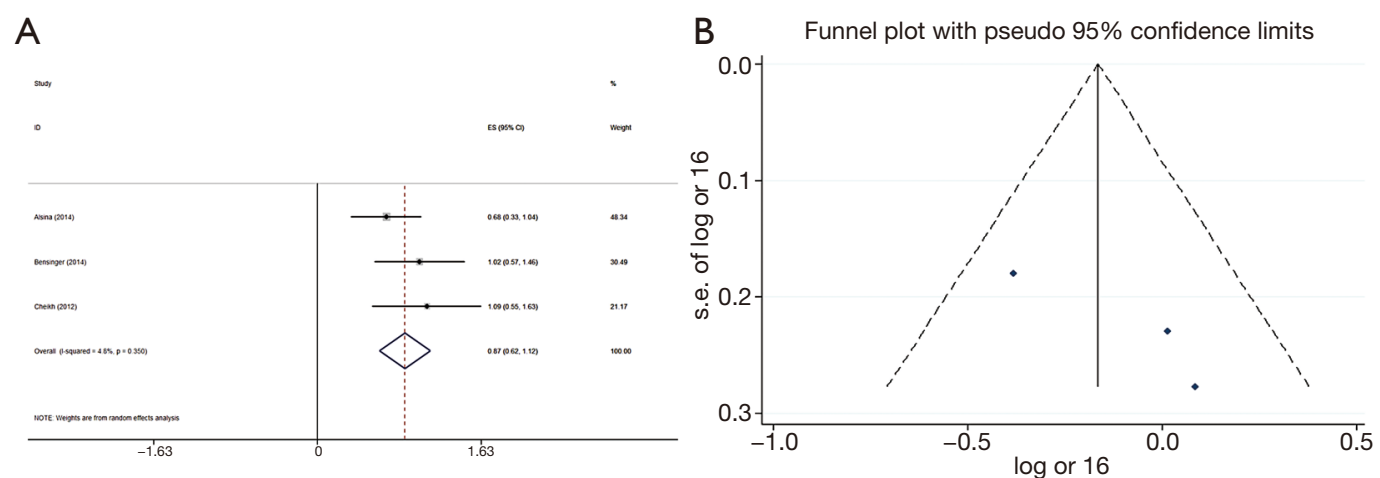

Figure 8 Meta-analysis of pain in patients with MM treated with lenalidomide. (A) Forest diagram and (B) Funnel diagram are the results of the analysis of the incidence of pain. MM, multiple myeloma.
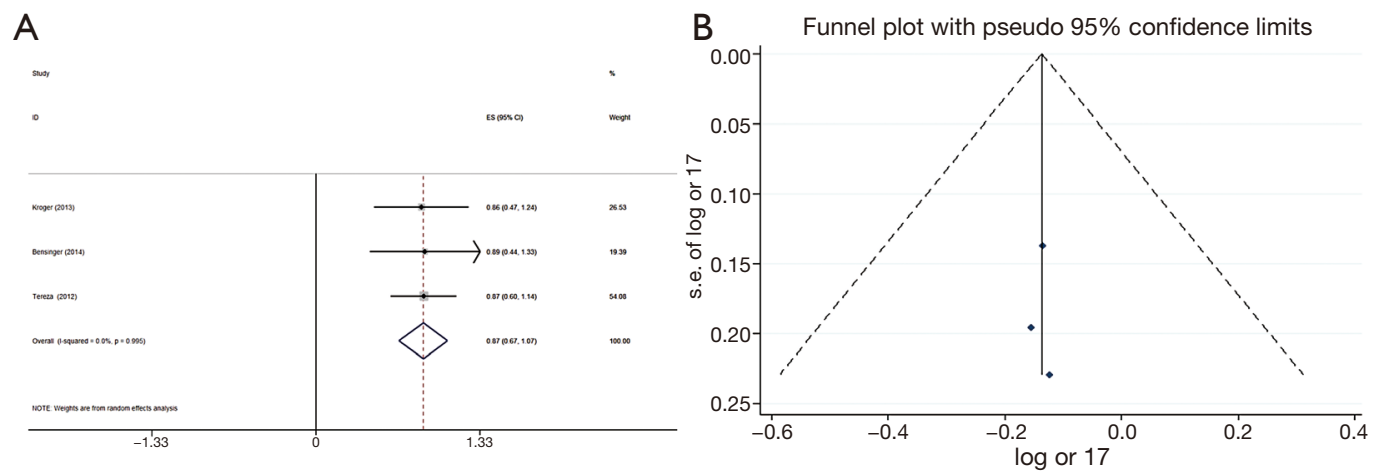

Figure 9 A meta-analysis of peripheral neuropathy in patients with MM treated with lenalidomide. (A) Forest diagram and (B) Funnel diagram are the analysis results of the prevalence of peripheral neuropathy. MM, multiple myeloma.

compared to the previous generation of thalidomide. In summary, lenalidomide has a good effect in the maintenance treatment of MM patients who have already received alloHSCT. Lenalidomide has a certain effect on reducing the incidence of infection and peripheral neuropathy, but no effect in controlling the postoperative GVHD, and there was no significant improvement in adverse reactions of the blood system. 

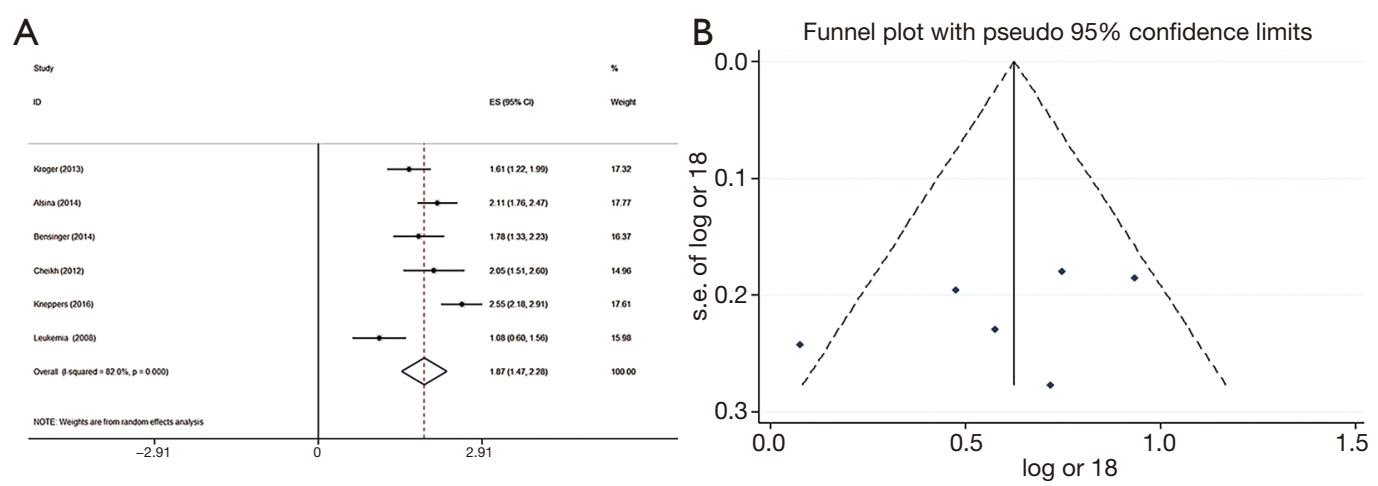

Figure 10 A meta-analysis of OS ( $\geq 2$ years) in patients with $M M$ after receiving lenalidomide treatment. (A) Forest diagram and (B) Funnel diagram are the results of OS analysis. MM, multiple myeloma; OS, overall survival.
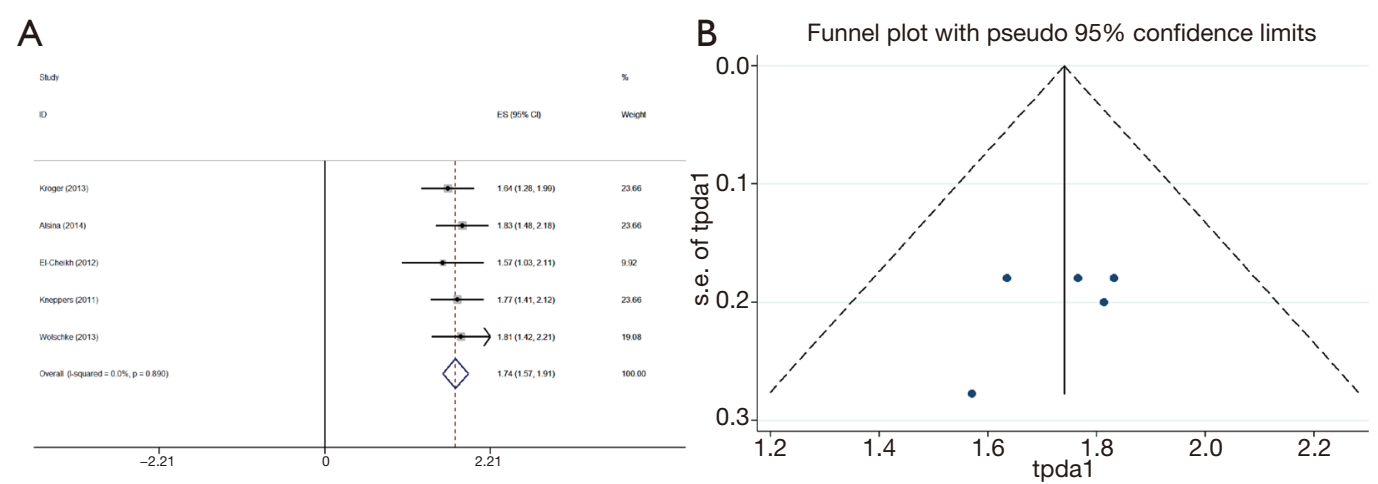

Figure 11 A meta-analysis of PFS ( $\geq 2$ years) in patients with MM after receiving lenalidomide treatment. (A) Forest diagram and (B) Funnel diagram are the results of PFS analysis. MM, multiple myeloma; PFS, progression-free survival.

Only 8 clinical studies were included in this metaanalysis, and the number of participants involved in each study was not large, resulting in a low sample size overall in this analysis, which reflected the current lack of clinically related studies. In the analysis of adverse reactions, there were only 3 studies included in the analysis of pain, infection, and peripheral neuropathy. This made our conclusions more sensitive, and we need to continue to include studies as they become available to reduce the sensitivity of the analysis results. Besides, since most of the included studies did not elaborate on the dosage and treatment duration of lenalidomide, the analysis did not control the variables for the dosage and period. This may have been one of the sources of heterogeneity in the analysis. The participants in this analysis were all Caucasian, so whether our analysis results would be applicable among other races requires verification.

In summary, this research confirmed that lenalidomide is effective in the treatment of MM patients after allo-HSCT, and reducing the incidence of infection and peripheral neuropathy, but it is not effective in reducing GVHD and blood system adverse reactions.

\section{Acknowledgments}

Funding: None.

\section{Footnote}

Reporting Checklist: The authors have completed the PRISMA reporting checklist. Available at https://dx.doi. org/10.21037/apm-21-1598

Conflicts of Interest: All authors have completed the ICMJE uniform disclosure form (available at https://dx.doi. org/10.21037/apm-21-1598). The authors have no conflicts 
of interest to declare.

Ethical Statement: The authors are accountable for all aspects of the work in ensuring that questions related to the accuracy or integrity of any part of the work are appropriately investigated and resolved.

Open Access Statement: This is an Open Access article distributed in accordance with the Creative Commons Attribution-NonCommercial-NoDerivs 4.0 International License (CC BY-NC-ND 4.0), which permits the noncommercial replication and distribution of the article with the strict proviso that no changes or edits are made and the original work is properly cited (including links to both the formal publication through the relevant DOI and the license). See: https://creativecommons.org/licenses/by-nc-nd/4.0/.

\section{References}

1. Rajkumar SV, Dimopoulos MA, Palumbo A, et al. International Myeloma Working Group updated criteria for the diagnosis of multiple myeloma. Lancet Oncol 2014;15:e538-48.

2. Xu P, Zhou R, Xu J, et al. Higher single dose of bortezomib plus thalidomide and dexamethasone is a promising therapy for newly diagnosed multiple myeloma. Transl Cancer Res 2019;8:2099-106.

3. Alexanian R, Bonnet J, Gehan E, et al. Combination chemotherapy for multiple myeloma. Cancer 1972;30:382-9.

4. Kröger N, Zabelina T, Klyuchnikov E, et al. Toxicity-reduced, myeloablative allograft followed by lenalidomide maintenance as salvage therapy for refractory/relapsed myeloma patients. Bone Marrow Transplant 2013;48:403-7.

5. Alsina M, Becker PS, Zhong X, et al. Lenalidomide

Cite this article as: Zhong J, Zhang X, Liu M. The efficacy and safety of lenalidomide in the treatment of multiple myeloma patients after allo-hematopoietic stem-cell transplantation: a systematic review and meta-analysis. Ann Palliat Med 2021;10(7):7736-7746. doi: 10.21037/apm-21-1598 maintenance for high-risk multiple myeloma after allogeneic hematopoietic cell transplantation. Biol Blood Marrow Transplant 2014;20:1183-9.

6. Bensinger WI, Green DJ, Burwick N, et al. A prospective study of lenalidomide monotherapy for relapse after AlloSCT for multiple myeloma. Bone Marrow Transplant 2014;49:492-5.

7. El-Cheikh J, Crocchiolo R, Furst S, et al. Lenalidomide plus donor-lymphocytes infusion after allogeneic stemcell transplantation with reduced-intensity conditioning in patients with high-risk multiple myeloma. Exp Hematol 2012;40:521-7.

8. Kneppers E, van der Holt B, Kersten MJ, et al. Lenalidomide maintenance after nonmyeloablative allogeneic stem cell transplantation in multiple myeloma is not feasible: results of the HOVON 76 Trial. Blood 2011;118:2413-9.

9. Wolschke C, Stübig T, Hegenbart U, et al. Postallograft lenalidomide induces strong NK cell-mediated antimyeloma activity and risk for $\mathrm{T}$ cell-mediated GvHD: Results from a phase I/II dose-finding study. Exp Hematol 2013;41:134-142.e3.

10. Minnema MC, van der Veer MS, Aarts T, et al. Lenalidomide alone or in combination with dexamethasone is highly effective in patients with relapsed multiple myeloma following allogeneic stem cell transplantation and increases the frequency of CD4+Foxp3+ T cells. Leukemia 2009;23:605-7.

11. Coman T, Bachy E, Michallet M, et al. Lenalidomide as salvage treatment for multiple myeloma relapsing after allogeneic hematopoietic stem cell transplantation: a report from the French Society of Bone Marrow and Cellular Therapy. Haematologica 2013;98:776-83.

(English Language Editor: J. Jones) 